\title{
HAWK 2.0: A Monte Carlo program for Higgs production in vector-boson fusion and Higgs strahlung at hadron colliders
}

\author{
Ansgar Denner ${ }^{\mathrm{a}}$, Stefan Dittmaier ${ }^{\mathrm{b}}$, Stefan Kallweit ${ }^{\mathrm{c}}$, Alexander Mück ${ }^{\mathrm{d}, *}$ \\ ${ }^{a}$ Universität Würzburg, Institut für Theoretische Physik und Astrophysik, \\ D-97074 Würzburg, Germany \\ ${ }^{b}$ Albert-Ludwigs-Universität Freiburg, Physikalisches Institut, \\ D-79104 Freiburg, Germany \\ ${ }^{c}$ Johannes Gutenberg-Universität Mainz, Institut für Physik, \\ PRISMA Cluster of Excellence, \\ D-55099 Mainz, Germany \\ ${ }^{d}$ RWTH Aachen University, Institut für Theoretische Teilchenphysik und Kosmologie, \\ D-52056 Aachen, Germany
}

\begin{abstract}
The Monte Carlo integrator HAWK provides precision predictions for Higgs production at hadron colliders in vector-boson fusion and Higgs strahlung, i.e.

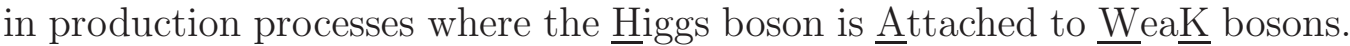
The fully differential predictions include the full QCD and electroweak nextto-leading-order corrections. Results are computed as integrated cross sections and as binned distributions for important hadron-collider observables.

Keywords: Higgs physics, radiative corrections, Monte Carlo integration
\end{abstract}

\section{PROGRAM SUMMARY}

Manuscript Title: HAWK 2.0: A Monte Carlo program for Higgs production in vector-boson fusion and Higgs strahlung at hadron colliders

Authors: Ansgar Denner, Stefan Dittmaier, Stefan Kallweit, Alexander Mück

Program Title: HAWK, version 2.0

\footnotetext{
${ }^{*}$ Corresponding author.

E-mail address: mueck@physik.rwth-aachen.de
} 
Journal Reference:

Catalogue identifier:

Licensing provisions: none

Programming language: Fortran 77, Fortran 90

Computer: Any computer with a Fortran 90 compiler

Operating system: Linux, Mac OS

RAM: less than $1 \mathrm{~GB}$

Keywords: Higgs physics, radiative corrections, Monte Carlo integration

Classification: 4.4 Feynman Diagrams, 11.1 General, High Energy Physics and Computing, 11.2 Phase Space and Event Simulation.

External routines/libraries: LHAPDF

(https://www.hepforge.org/downloads/lhapdf).

Nature of problem: Precision calculation of cross sections and differential distributions for Higgs-boson production in vector-boson fusion and Higgs strahlung at the LHC as described in Refs. [1 3].

Solution method: Multi-channel Monte Carlo integration of perturbative matrix elements including higher-order QCD and electroweak corrections which are based on a Feynman-diagrammatic calculation.

Restrictions: For vector-boson fusion, only the Higgs-boson decay into a pair of massless singlets is supported. For Higgs strahlung, decay products are not supported, while the Higgs boson can be off shell.

Running time: Meaningful results can be obtained within a few hours on a single core. The statistical uncertainty of the results typically improves with the square root of the run time.

[1] M. Ciccolini, A. Denner, S. Dittmaier, Phys. Rev. Lett. 99 (2007) 161803 arXiv:0707.0381 [hep-ph]].

[2] M. Ciccolini, A. Denner, S. Dittmaier, Phys. Rev. D77 (2008) 013002 arXiv:0710.4749 [hep-ph]].

[3] A. Denner, S. Dittmaier, S. Kallweit and A. Mück, JHEP 1203 (2012) 075 arXiv:1112.5142 [hep-ph]]. 


\section{Introduction}

After the discovery of the Higgs boson [4, 5], precision predictions for its production channels are of paramount importance. The measurement of the Higgs properties is and will continue to be one of the most important tasks in the physics programme at CERN's Large Hadron Collider (LHC) [69]. Searching for possible deviations from its properties as predicted by the Standard Model (SM) of particle physics requires precise predictions for the underlying measurements [10 12]. While the total Higgs production rate is dominated by the gluon-fusion process, Higgs production in vectorboson fusion (VBFH) and in association with a weak boson, also known as Higgs strahlung, play an important role in unveiling the nature of the Higgs boson. In this work, we present the Monte Carlo program HAWK [13] which provides precision predictions for these production channels, which are based on Refs. [1 3] . In particular, the electroweak (EW) corrections contained in the cross-section predictions for VBFH and VH compiled by the LHC Higgs Cross Section Working Group (LHCHXSWG) [6-9] were obtained with the HAWK program.

Higgs production in vector-boson fusion is experimentally accessed by measuring the Higgs decay products in association with two forward tagging jets having a large rapidity separation. Applying the corresponding cuts, as suggested in Refs. [14 18], the cross section is dominated by tree-level diagrams where two quark lines exchange a weak vector boson in the $t$ channel and the Higgs boson is emitted by the weak boson. The corresponding diagrams are shown in Fig. 1. At the same order in the weak coupling constant there are also $s$-channel diagrams. Their contribution to the cross section is suppressed if vector-boson fusion cuts are applied. In fact, the $s$-channel contribution corresponds to what is usually called the Higgs-strahlung process where the weak boson decays hadronically ( $\mathrm{pp} \rightarrow \mathrm{HV} \rightarrow \mathrm{H}+2$ jets). HAWK includes the full set of diagrams ( $s, t$, and $u$ channels), all interferences, and the corresponding next-to-leading order (NLO) QCD and EW corrections [1, 2]. Hence, the distinction between vector-boson fusion and Higgs strahlung with a hadronically decaying weak boson can be done in a completely physical way employing the corresponding experimental cuts.

The Higgs-strahlung process $\mathrm{pp} \rightarrow \mathrm{H} V$ is experimentally promising when the weak boson $V$ decays leptonically. The leptons provide trigger objects and allow for background suppression. The processes pp $\rightarrow \mathrm{HZ} \rightarrow \mathrm{H}^{+} l^{-}$, $\mathrm{pp} \rightarrow \mathrm{HZ} \rightarrow \mathrm{H} \bar{\nu}_{l} \nu_{l}, \mathrm{pp} \rightarrow \mathrm{HW}^{+} \rightarrow \mathrm{H} l^{+} \nu_{l}$, and $\mathrm{pp} \rightarrow \mathrm{HW}^{-} \rightarrow \mathrm{H}_{l} l^{-}$are also 

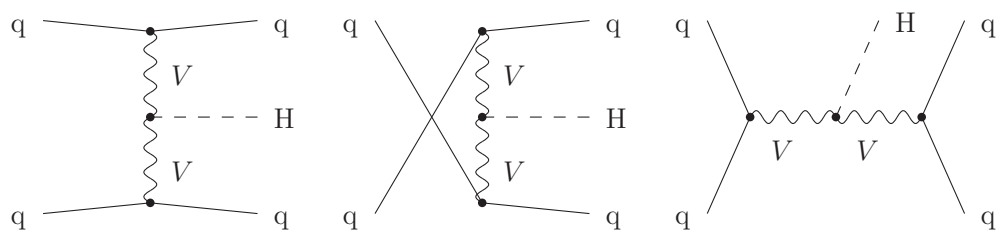

Figure 1: Leading-order diagrams for VBFH production. The diagrams correspond to the $t$-channel (left), $u$-channel (middle), and $s$-channel (right).

calculated by HAWK including the complete NLO QCD and EW corrections in the SM [3].

The theory predictions for the considered processes with respect to higher orders in QCD corrections are quite advanced. The total cross section for vector-boson fusion is known to NNLO QCD accuracy in the so-called DIS approximation [19, 20]. Differential vector-boson fusion at NLO QCD accuracy [21 24] was matched to parton showers [12, 25, 26]. The NLO QCD and EW corrections are also available in the alternative Monte Carlo program VBFNLO [24, 27], however, restricted to the $t$-channel approximation which is sufficient only if vector-boson fusion (VBF) cuts are applied.

The Higgs-strahlung process was calculated in NNLO QCD 28 31], and the dominant parts are now also available in a fully differential form [32, 33]. The NLO QCD prediction [34 is known for a long time, and is available in public programs [35, 36]. It was also matched to parton showers [37] and merged with matched NLO calculations for higher jet multiplicities [38]. The EW corrections to inclusive cross sections for Higgs strahlung were presented in Ref. [39]. The loop-induced process gg $\rightarrow \mathrm{ZH}$ and the corresponding NLO QCD corrections were calculated in the large- $m_{\mathrm{t}}$ approximation in Ref. [40].

HAWK provides the state-of-the-art tool to predict fully differential EW corrections [1 3] which can be combined via differential reweighting with the most advanced QCD predictions. The usual hadron-collider cuts are available and can be imposed via a simple input structure. The standard distributions are also available and provided via text files which are easy to process. Additional user-defined cuts and distributions are easy to implement, and support from the authors is provided on request.

Within HAWK, the complex-mass scheme [41, 42] is employed to calculate the EW corrections to the Higgs-strahlung processes with potentially resonant weak bosons. HAWK implements matrix elements which are calcu- 
lated in the traditional Feynman-diagrammatic approach [43 46] employing advanced techniques for tensor reduction to standard scalar integrals with complex masses [47 50]. It employs Monte Carlo techniques for phase-space integration which are detailed in Refs. [51, 52]. The combination of real and virtual pieces is based on dipole subtraction [53 55].

HAWK can be employed for proton-proton colliders like the LHC, but also proton-anti-proton colliders. As part of the full NLO EW corrections, HAWK provides predictions for partonic channels with incoming photons. Within HAWK, the decay of the Higgs boson into a pair of massless gauge singlets is supported for the VBFH channel. The Higgs boson can be produced on-shell, or different options for an off-shell Higgs propagator can be used. Effects of anomalous HZZ/HWW couplings can be included in the predictions. For VBFH, we also include leading heavy-Higgs-boson effects at two-loop order which, however, are negligible for the observed Higgs boson at $125 \mathrm{GeV}$.

This work is structured as follows. In Section 2, we highlight some details of HAWK in connection with the underlying physics. In Section 3, all the necessary information for the user is given how to run and use the HAWK program. Along with the practical information on all the user-defined input, we give some details on the program itself. Section 4 introduces the way HAWK provides the computed results. In Section 5, we make some concluding remarks.

\section{EW corrections and off-shell effects in HAWK}

Specific strengths of the predictions provided by the HAWK program concern the careful treatment of EW corrections and off-shell effects, as discussed in the following sections.

\section{1. $\alpha_{G_{\mathrm{F}}}$ scheme}

The $\alpha_{G_{F}}$ scheme is used as input-parameter scheme. The electromagnetic coupling constant is derived from the Fermi constant according to

$$
\alpha_{G_{\mathrm{F}}}=\frac{\sqrt{2} G_{\mathrm{F}} M_{\mathrm{W}}^{2}}{\pi}\left(1-\frac{M_{\mathrm{W}}^{2}}{M_{\mathrm{Z}}^{2}}\right) .
$$

This procedure takes into account some higher-order effects already at tree level. 


\subsection{Complex-mass scheme and input masses}

Gauge-boson resonances are treated using the complex-mass scheme [42], i.e. all corresponding decay and off-shell effects are supported in NLO accuracy in the full phase space. The program reads the on-shell masses and widths and translates them internally to the pole masses and widths (see Section 3.3.2). The latter are then used in propagators, the complex weak mixing angle, and other derived couplings.

\subsection{Final-state photons}

Both in the $\mathrm{VBFH}$ process and in $\mathrm{VH}$ production, the potential appearance of an additional photon (as part of the real photonic EW corrections) requires particular attention in the definition of infrared(IR)-safe observables (see also Section 3.3.3).

In $\mathrm{VBFH}$ production, where two hard jets and a photon appear in the final state of the real NLO EW correction, IR-safety is achieved in HAWK upon treating the photon as any other parton in the jet algorithm when a photon-jet pair becomes unresolved. In case the photon and a jet are merged, the resulting object is treated as a jet.

In $V \mathrm{H}$ production, where the decay of $V$ produces at least one charged lepton, IR issues occur when the photon becomes collinear to a charged lepton. If the lepton is a muon, HAWK admits a perfect isolation of the photon from the muon, leading to final-state-radiation corrections that are enhanced by a logarithm $\alpha \ln \left(m_{\mu} / Q\right)$ of the muon mass $m_{\mu}$ over some large scale $Q$. Technically, the lepton-photon isolation is performed according to Ref. [54]. If the lepton is an electron or positron, collinear photons and electrons/positrons appear as one electromagnetic shower in the detector, so that photon and lepton are recombined to a single quasi-particle when they become sufficiently collinear. After this photon recombination no logarithmically enhanced final-state-radiation effects remain.

\subsection{Combination of EW corrections from HAWK with external QCD-based predictions}

Apart from producing predictions with full NLO QCD+EW accuracy, HAWK can be used to calculate differential EW correction factors for any one- or more-dimensional distribution $\mathrm{d} \sigma / \mathrm{d} \mathcal{O}$, which can be used as differ-

ential EW reweighting factors to improve other QCD-based predictions for 
VBFH or $V H$ production that go beyond NLO accuracy. We suggest to use

$$
\frac{\mathrm{d} \sigma_{\mathrm{QCD} \times \mathrm{EW}}^{\text {best }}}{\mathrm{d} \mathcal{O}}=\left[1+\delta_{\mathrm{EW}}(\mathcal{O})\right] \frac{\mathrm{d} \sigma_{\mathrm{QCD}}^{\text {best }}}{\mathrm{d} \mathcal{O}}+\frac{\mathrm{d} \sigma_{\gamma}}{\mathrm{d} \mathcal{O}},
$$

i.e. to assume approximate factorization for $\mathrm{QCD}$ and $\mathrm{EW}$ corrections. In the above formula, $\mathrm{d} \sigma_{\mathrm{QCD}}^{\text {best }}$ is the best available QCD prediction to be obtained, for example, by one of the QCD calculations mentioned in the introduction. HAWK provides the differential EW corrections $\delta_{\mathrm{EW}}(\mathcal{O})$ and the differential cross section $\mathrm{d} \sigma_{\gamma}$ due to photons in the initial state (see Section 4.1). In particular, the total cross sections reported in Refs. 10 12] by the LHCHXSWG were obtained in this way. Note that EW corrections provided by HAWK are almost independent of the PDF set which is used in the calculation. Note also that HAWK provides all results in form of binned distributions. Unweighted events are not available.

\subsection{Higgs-boson decays}

The current version of HAWK optionally supports only the Higgs-boson decay channel into a pair of massless singlets without any correction to this decay. The corresponding branching ratio has to be provided as external input for the specific Higgs-boson decay (see Section 3.3.5). Note that the decay into two photons is well approximated in HAWK by the singlet decay. Since there are no corrections due to real radiation to this decay at NLO, the (purely virtual) EW corrections can be included by the appropriate $\mathrm{H} \rightarrow \gamma \gamma$ branching ratio.

\section{The usage of HAWK}

\subsection{HAWK installation}

HAWK is a stand-alone Fortran 77/90 code [13]. No additional libraries are needed to run HAWK, apart from the LHAPDF library [56] to employ up-to-date PDF sets, as discussed below. It has been tested under various operating systems, including different Linux distributions and MAC OS without problems. It should also be compilable with any standard Fortran compilers and has been successfully tested using GNU Fortran (GCC) 4.1.2, 4.4.7, 4.6.3, 4.7.2, pgf95 10.1-0, Intel Fortran Compiler 11.1, 13.1, and Openmpi 1.6.4.

For installation, gunzip and untar HAWK-2.0.tar.gz which will unpack into the directory ./HAWK-2.0. The source files are contained in 
the HAWK-2.0/src directory. The directory HAWK-2.0/sampleruns contains input files and the corresponding results of sample runs. The directories HAWK-2.0/bin, HAWK-2.0/obj, and HAWK-2.0/mod are for the executable, object files, and module files, respectively. To select the compiler or compiler options, the Makefile can be edited. To compile HAWK, simply go to the HAWK-2.0 directory and issue make in the command line to generate the executable hawk-2.0.

For usage of up-to-date parton distributions functions (PDFs), an interface to the LHAPDF library [56] is provided. The LHAPDF library is not part of the HAWK distribution. In order to use LHAPDF (which is the default option), the preprocessor flag includeLHAPDF must be set in the Makefile, and the corresponding library must be provided. This can be done by setting the variable LHAPDF_CONFIG to the executable lhapdf-config of the LHAPDF version that should be used. Alternatively, the path to the LHAPDF library and, if needed, include files can be set manually via LHAPDF and LHAPDFFFLAGS, respectively. For usage without LHAPDF, only the code and tables for two PDF sets (MRST2004QED and CTEQ6) are included in the distribution.

\subsection{HAWK execution}

To run HAWK, an input file from the standard input is needed (see, however, the remarks for parallel execution using mpi in Section 3.4). The program can be executed using:

$$
\text { . /hawk-2.0 < inputfile }
$$

Output will be written to standard output. If an output file is specified in the input file, it will be used to store the central results.

All input is delivered via the inputfile. This has to be specified via standard input, otherwise HAWK does not start. Its general format can be seen from the default inputfile input_default in the directory HAWK-2.0/sampleruns and the input files of the subdirectories. While the sample input files specify all relevant parameters, it is sufficient to specify those values that differ from the default. As a general remark, do not forget the "d0" after "double precision" quantities.

When using LHAPDF, the relevant files for the PDFs should be in the directory PDFsets of the LHAPDF installation (see Section 3.3.6). When using the included PDF sets, the corresponding table files (qed6-10gridp.dat 
for MRST2004QED or cteq6m.tbl, cteq61.tbl, cteq611.tbl for CTEQ6) must be available in the working directory where HAWK is executed.

\subsection{HAWK input}

The detailed description of HAWK in the following sections follows the structure of the default input file input_default which is provided with the HAWK distribution. All supported options for a given user input are detailed, and the underlying physics is briefly explained.

\subsubsection{Global parameters}

outputfile: a character string that specifies the name of the output file.

$$
\begin{aligned}
\text { outputfile=', } & \text { For a blank character string (default) all } \\
& \text { output is written to standard output. } \\
\text { outputfile='filename' : } & \text { Any other string usable as a file name } \\
& \text { redirects the output to a file with the } \\
& \text { given filename. }
\end{aligned}
$$

selprocess: integer that selects the process to be calculated.

selprocess $=0: p p \rightarrow$ jjH production $(\mathrm{VBFH})$ (default),

selprocess=1: pp $\rightarrow l^{+} \nu_{l} \mathrm{H}$ production $\left(\mathrm{W}^{+} \mathrm{H}\right)$,

selprocess $=2: \mathrm{pp} \rightarrow \bar{\nu}_{l} l^{-} \mathrm{H}$ production $\left(\mathrm{W}^{-} \mathrm{H}\right)$,

selprocess=3: pp $\rightarrow l^{+} l^{-} \mathrm{H}$ production $(\mathrm{ZH})$,

selprocess $=4: \mathrm{pp} \rightarrow \bar{\nu}_{l} \nu_{l} \mathrm{H}$ production $(\mathrm{ZH})$.

A change of this flag sets all options and cuts to their respective default values. They can be changed thereafter. In particular, for VBFH, the typical VBFH cuts are selected. To use HAWK for Higgs strahlung with hadronic vector-boson decay, one has to select selprocess=0 and change the respective kinematic cuts appropriately thereafter.

nevents: integer that selects the number of generated weighted events.

nevents $=10000000$ : is the default value.

The number of weighted events should be at least $10^{7}$ to guarantee that the multi-channel integration yields reliable results and error estimates. For histograms more events should be generated. For the published distributions we used $10^{9}$ events. 
energy: double-precision number that selects the centre-of-mass energy in the $\mathrm{pp} / \mathrm{p} \overline{\mathrm{p}}$ system in $\mathrm{GeV}$.

energy $=14000 \mathrm{~d} 0$ : is the default value.

sppbar: integer that selects the hadron collider type.

sppbar $=0$ : pp collider such as the LHC (default), sppbar $=1: p \bar{p}$ collider such as the Tevatron.

For a $\mathrm{p} \overline{\mathrm{p}}$ collider, the proton $\mathrm{p}$ is going in the $+z$ direction.

\subsubsection{Input parameters}

The following input parameters can be specified with double-precision values. Here, we also state the corresponding default values:

$\begin{array}{ll}\mathrm{gf}=0.1166370 \mathrm{~d}-04 & : \text { Fermi constant } G_{\mathrm{F}} \text { in } \mathrm{GeV}^{-2}, \\ \mathrm{mz}=91.1876 \mathrm{~d} 0 & : \text { Z-boson mass } M_{\mathrm{Z}} \text { in } \mathrm{GeV}, \\ \mathrm{gz}=2.4952 \mathrm{~d} 0 & : \text { Z-boson width } \Gamma_{\mathrm{Z}} \text { in } \mathrm{GeV}, \\ \mathrm{mw}=80.398 \mathrm{~d} 0 & : \text { W-boson mass } M_{\mathrm{W}} \text { in } \mathrm{GeV}, \\ \mathrm{gw}=2.08872 \mathrm{~d} 0 & : \text { W-boson width } \Gamma_{\mathrm{W}} \text { in } \mathrm{GeV}, \\ \mathrm{mmu}=0.105658367 \mathrm{~d} 0 & : \text { muon mass } m_{\mu} \text { in } \mathrm{GeV}, \\ \mathrm{mt}=172.5 \mathrm{~d} 0 & : \text { top-quark mass } m_{\mathrm{t}} \text { in } \mathrm{GeV}, \\ \mathrm{mh}=126.0 \mathrm{~d} 0 & : \text { Higgs-boson mass } M_{\mathrm{H}} \text { in } \mathrm{GeV}, \\ \mathrm{gh}=4.21 \mathrm{~d}-3 & : \text { Higgs-boson width } \Gamma_{\mathrm{H}} \text { in } \mathrm{GeV}, \\ \text { sinthetac=0.225d0 } & : \text { sine of the Cabibbo angle } \theta_{\mathrm{c}} .\end{array}$

For the weak gauge bosons $V=\mathrm{W}, \mathrm{Z}$, the program reads the on-shell masses and widths and translates them internally to the pole masses and widths,

$$
\begin{aligned}
M_{V}^{\text {pole }} & =M_{V}^{\text {on-shell }} / \sqrt{1+\left(\Gamma_{V}^{\text {on-shell }} / M_{V}^{\text {on-shell }}\right)^{2}}, \\
\Gamma_{V}^{\text {pole }} & =\Gamma_{V}^{\text {on-shell }} / \sqrt{1+\left(\Gamma_{V}^{\text {on-shell }} / M_{V}^{\text {on-shell }}\right)^{2}} .
\end{aligned}
$$

These values are then used in propagators, the complex weak mixing angle, and other derived couplings. The output provides the pole masses, not the input masses.

The masses of the light fermions appear internally, but in the $\alpha_{G_{F}}$ scheme used the results are practically independent of the specific values. Only for bare leptons in the final state of the Higgs-strahlung process their masses 
become relevant. Here, HAWK uses the input value for the muon mass $\mathrm{mmu}$, as the muon case is the only one where the bare-lepton treatment is reasonable.

The CKM matrix is always treated as real and block-diagonal, i.e. the first two generations mix with each other, but not with the third generation. In this approximation, the CKM matrix is fully specified by the Cabibbo angle.

\subsubsection{Recombination}

IR-safe observables are constructed by properly defining jets in QCD. For $\mathrm{VBFH}$, the recombination of quarks and gluons into jets is performed according to the $k_{\mathrm{T}}$ algorithm [57]. The different variants of the employed jet algorithm are discussed below (see ktpower). Including EW corrections, also photons are treated as partons in the jet algorithm for VBFH.

ktpower: integer that selects the type of $k_{\mathrm{T}}$ algorithm.

ktpower=1 : standard $k_{\mathrm{T}}$ algorithm, ktpower $=0$ : Cambridge-Aachen algorithm, ktpower $=-1$ : anti- $k_{\mathrm{T}}$ algorithm.

dparameter $=0.8 \mathrm{~d} 0:$ double-precision number that sets the $D$ parameter of the jet algorithm, i.e. the jet size ( $0.8 \mathrm{~d} 0$ is the default value).

etacut (parton) $=5.0 \mathrm{~d} 0$ : double-precision number that specifies the pseudo-rapidity cut on partons in the jet algorithm (5.0d0 is the default value). Partons that do not pass the cut are not considered for recombination in the jet algorithm.

For $\mathrm{WH} / \mathrm{ZH}$, there are no jets in the final state at LO, and at NLO there is at most one jet or photon, so that no jet algorithm is applied here. However, the recombination of charged leptons and a bremsstrahlung photon is important if no perfect isolation of leptons can be achieved, as e.g. for electrons and photons which appear as showers in the electromagnetic calorimeters of the detectors.

sbarelep: integer that steers lepton-photon isolation.

sbarelep=1 : leptons are isolated from collinear photons (default), sbarelep=0 : no such isolation is applied. 
Technically, the lepton-photon isolation is performed according to Ref. [54]. As lepton-photon isolation makes sense only in case of muons in the final state, the lepton mass in the splittings is automatically set to the muon mass. To investigate the electron final state, sbarelep $=0$ is recommended. In this case, leptons and photons are recombined:

dgammaparameter $=0.1 \mathrm{~d} 0$ : double-precision number that specifies the $D$ parameter of lepton-photon recombination (0.1d0 is the default value).

For $R_{\gamma l}<D$, the lepton and the photon are recombined. If this holds for more than one lepton, the one with the smaller $R_{\gamma l}$ is recombined with the photon. The recombined particle, which carries the total momentum of the two com-

bined particles, is treated as a lepton. Here, $R_{\gamma l}=\sqrt{\left(y_{l}-y_{\gamma}\right)^{2}+\left(\varphi_{l}-\varphi_{\gamma}\right)^{2}}$ is the usual separation in rapidity $y$ and angle $\varphi$ in the transverse plane.

For other recombination schemes the subroutines vbfh_recombination in vbfh_public.F or whzh_recombination in whzh_public.F have to be modified.

\subsubsection{Cuts}

The cuts on final-state jets or leptons can be set by a flag to predefined values, but can also be chosen individually.

scuts : integer that governs the phase-space cuts.

The meaning of scuts depends on the chosen value for selprocess. For VBFH, i.e. selprocess=0, the possible values correspond to:

scuts=1 : standard cuts as defined in Refs. [1, 2] (see also Ref. [58]), jets are ordered according to $p_{\mathrm{T}}$ of jets (default),

scuts=2 : standard cuts as defined in Refs. [1, 2] (see also Ref. [58]), jets are ordered according to the energy of jets, scuts $=0$ : no cuts (only useful for total cross sections).

For Higgs strahlung, i.e. selprocess $=1,2,3,4$, the possible values correspond to:

scuts=1 : standard cuts for detected leptons as defined in Ref. [3] (default),

scuts=2 : standard cuts for undetected leptons as defined in Ref. [3], scuts=0: no cuts (only useful for total cross section). 
For VBFH, according to the choice for scuts, in the following the leading jet $j_{1}$ is the jet with the largest transverse momentum or largest energy. The next-to-leading jet $j_{2}$ is the jet with the second largest transverse momentum or second largest energy, etc.

The above options set various cuts to their default values which are stated below. They can be changed thereafter. For other cut schemes the subroutines applycut or applyhiggscut in public.F or applyjetcut in vbfh_public.F or applylepcut or applylephiggscut in whzh_public.F have to be modified.

All the standard cut parameters can be set/changed after choosing scuts $>0$. In the following the needed input is given together with the default values which depend on scuts and selprocess. All the following cuts are specified by double-precision numbers, and dimensionful quantities are given in $\mathrm{GeV}$. For VBFH, i.e. selprocess=0, the standard cuts are as follows:

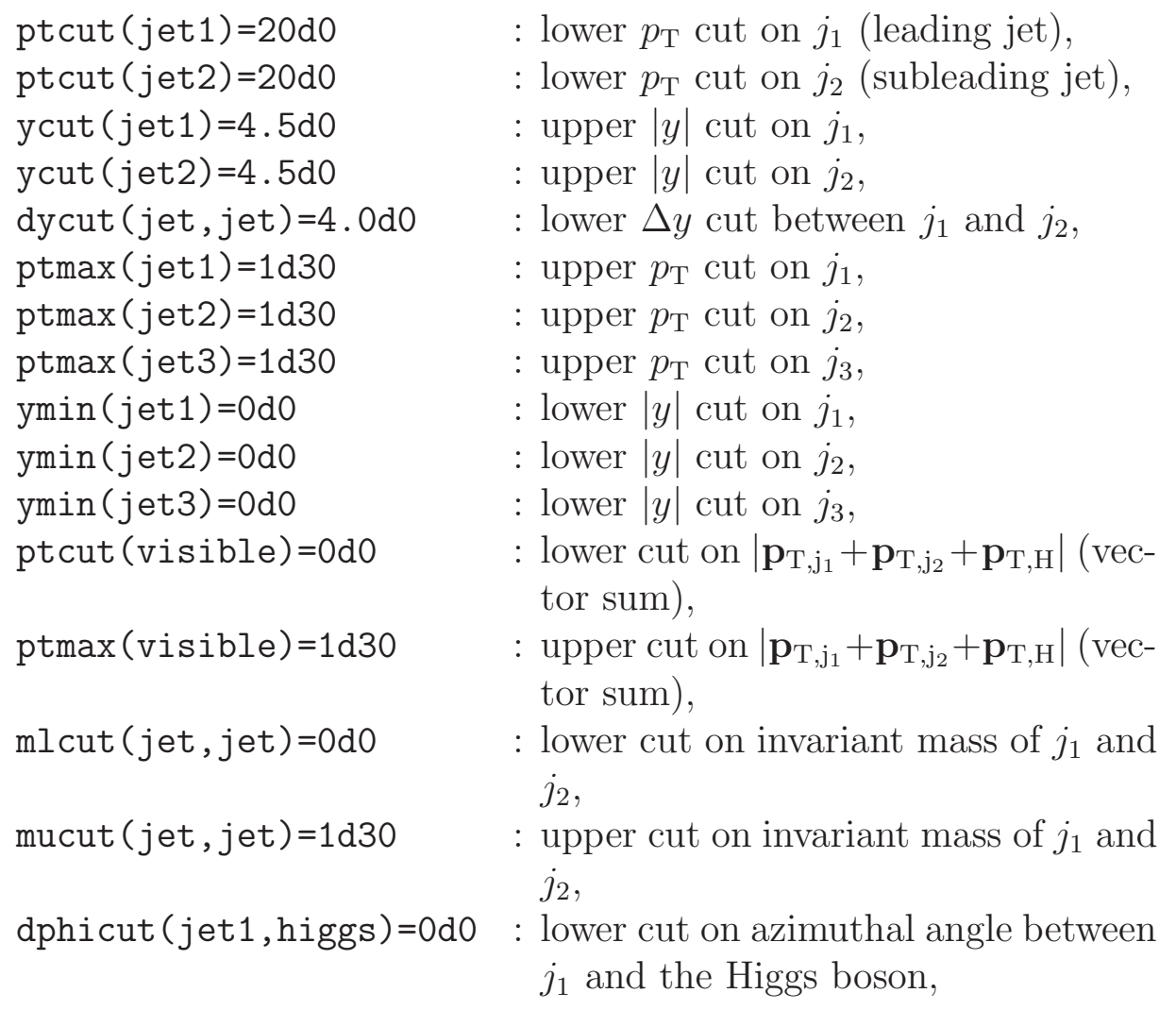




$$
\begin{array}{ll}
\text { dphicut (jet2, higgs)=0d0 } & : \text { lower cut on azimuthal angle between } \\
& j_{2} \text { and the Higgs boson, } \\
\text { ptcut (higgs) }=0 \mathrm{~d} 0 & : \text { lower } p_{\mathrm{T}} \text { cut on the Higgs boson, } \\
\text { ycut }(\text { higgs })=1 \mathrm{~d} 10 & : \text { upper }|y| \text { cut on the Higgs boson, } \\
\text { ecut }(\text { higgs })=0 \mathrm{~d} 0 & : \text { lower energy cut on the Higgs boson, } \\
\text { mlcut (higgs) }=1 \mathrm{~d}-2 & : \text { lower cut on the invariant mass of an } \\
& \text { off-shell Higgs boson, } \\
\text { mucut (higgs })=1 \mathrm{~d} 30 & : \text { upper cut on the invariant mass of an } \\
& \text { off-shell Higgs boson. }
\end{array}
$$

In the above, $y$ denotes the rapidity of the specified final-state object and $p_{\mathrm{T}}$ its transverse momentum. In particular, the lower cut on the rapidity difference of the leading jets, dycut (jet, jet) $=4.0 \mathrm{~d} 0$, is specific to select the VBFH process. There is one more VBFH-specific cut:

hemispherecut : integer that governs the condition $y\left(j_{1}\right) y\left(j_{2}\right)<0$.

hemispherecut=1 : $y\left(j_{1}\right) y\left(j_{2}\right)<0$ is required for $j_{1}$ and $j_{2}$ (default), hemispherecut $=0: y\left(j_{1}\right) y\left(j_{2}\right)<0$ cut is not applied.

To implement a jet veto, ptmax and ymin can be used in combination to define final states without detectable jets, i.e. a jet is considered undetectable if either $p_{\mathrm{T}}($ jet $)>\operatorname{ptmax}($ jet $)$ or $\mid y($ jet $) \mid<\operatorname{ymin}($ jet $)$ fails. The cuts for $j_{1}$ apply to all jets, those for $j_{2}$ for all but the leading jet, those for $j_{3}$ only veto a third jet. Set $\operatorname{ptmax}($ jet 1$)=1 \mathrm{~d} 30$ and $/$ or $\operatorname{ymin}($ jet 1$)=0 \mathrm{~d} 0$ to switch off these cuts, and likewise for $j_{2}$ and $j_{3}$.

For $\mathrm{WH} / \mathrm{ZH}$, i.e. selprocess=1,2,3,4, the standard cuts partly depend on the value of scuts. For scuts=1, they are given by:

$$
\begin{array}{ll}
\operatorname{ptcut}(l e p)=20 \mathrm{~d} 0 & : \text { lower } p_{\mathrm{T}} \text { cut on leptons, } \\
\operatorname{ycut}(l e p)=2.5 \mathrm{~d} 0 & : \text { upper }|y| \text { cut on leptons, } \\
\operatorname{ptmax}(l e p)=1 \mathrm{~d} 10 & : \text { upper } p_{\mathrm{T}} \text { cut on leptons, } \\
\operatorname{ymin}(l e p)=0 \mathrm{~d} 0 & : \text { lower }|y| \text { cut on leptons. }
\end{array}
$$

For scuts=2, they are given by:

$$
\begin{array}{ll}
\operatorname{ptcut}(l e p)=0 \mathrm{~d} 0 & : \text { lower } p_{\mathrm{T}} \text { cut on leptons, } \\
\operatorname{ycut}(l e p)=1 \mathrm{~d} 10 & : \text { upper }|y| \text { cut on leptons, } \\
\operatorname{ptmax}(l e p)=20 \mathrm{~d} 0 & : \text { upper } p_{\mathrm{T}} \text { cut on leptons, } \\
\operatorname{ymin}(l e p)=2.5 \mathrm{~d} 0 & : \text { lower }|y| \text { cut on leptons. }
\end{array}
$$


The default cuts, which are the same for $\operatorname{scuts=1}$ and scuts=2, are given by:

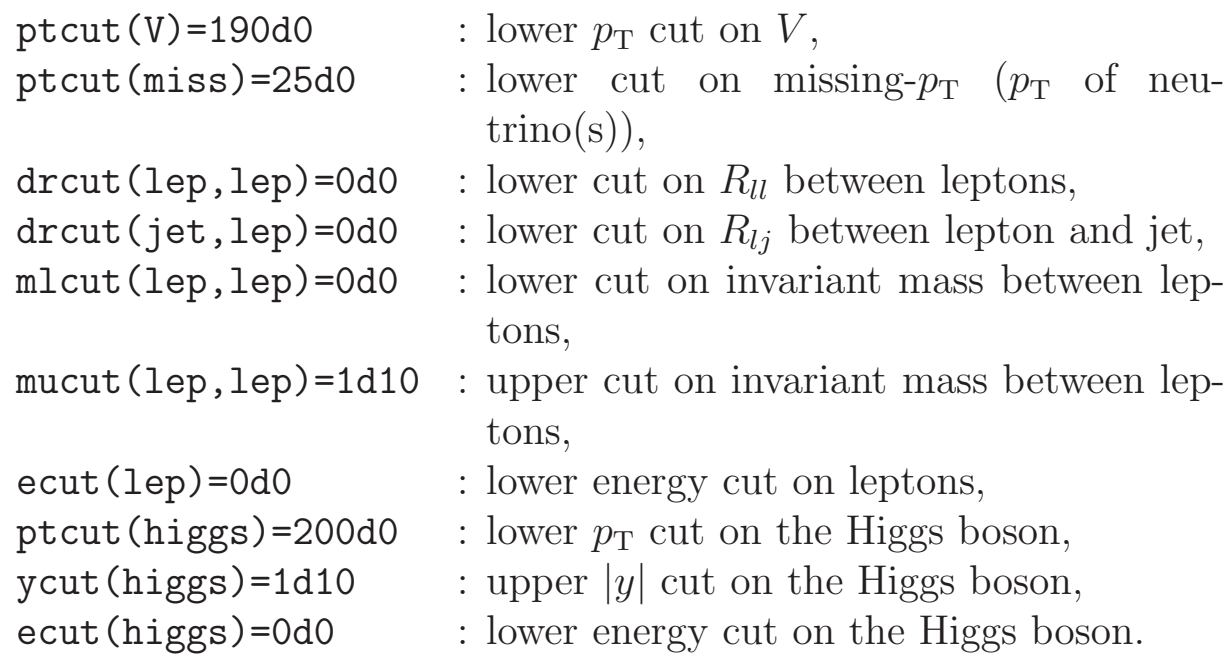

In analogy to a jet veto, ptmax and ymin are used in combination to define final states without detectable leptons, i.e. a lepton is considered undetectable if either $p_{\mathrm{T}}(l)>\operatorname{ptmax}(l e p)$ or $|y(l)|<\operatorname{ymin}(l e p)$ fails. Set $\operatorname{ptmax}(l e p)=1 \mathrm{~d} 10$ and/or $y m i n(l e p)=0 \mathrm{~d} 0$ to switch off this cut. These cuts are particularly useful to calculate the contribution of the $\mathrm{pp} \rightarrow \mathrm{H} l^{+} \nu_{l}$ and $\mathrm{pp} \rightarrow \mathrm{H} \bar{\nu}_{l} l^{-}$processes to the $\mathrm{H} \bar{\nu}_{l} \nu_{l}$ final state, i.e. a Higgs boson plus missing $p_{\mathrm{T}}$, due to a missed charged lepton.

\subsubsection{Off-shell Higgs boson, Higgs decays and corresponding cuts}

Within HAWK, there are several options that allow to choose between on-shell and off-shell Higgs-boson production and to specify the details of the off-shell treatment.

shtr : integer that selects on-shell or off-shell Higgs production.

shtr=0 : on-shell Higgs boson (default),

shtr=1 : off-shell Higgs boson,

shtr=2 : off-shell Higgs boson decaying into a pair of massless singlets (for VBFH, i.e. selprocess=0, only).

For shtr=1 the external Higgs boson is off shell, and the resonance is treated according to the flag shbw (see below). For shtr=2 additionally an isotropic Higgs decay into a pair of massless scalar singlets is included. This can be 
used to mimic any two-body decay into massless particles, e.g. the decay into photons. For shtr=1 or shtr=2 the width of the Higgs boson has to be given as input, or the width can be calculated internally (see gh and sgh below). For shtr=2, in addition, the branching ratio for the decay into singlets has to be specified. For an off-shell Higgs boson the EW corrections are calculated after an on-shell projection of the momenta (required by gauge invariance).

The following flags dictate the details of the off-shell treatment:

shbw : integer that determines the treatment of the off-shell Higgs propagator for $\operatorname{shtr}=1$ or $\operatorname{shtr}=2$.

shbw=0 : standard Breit-Wigner with mass mh and (constant) width gh,

shbw=1 : off-shell propagator according to Ref. [59], Eq. (4.6) (default if shtr $\neq 0$ ). Note that choosing the flag $s g h=0$ is not allowed in this case.

sgh : integer that determines the treatment of the Higgs-boson width. sgh=0 : use Higgs-boson width as specified in input by gh, sgh=1 : gh is set for the input Higgs mass using an interpolation of the results in Ref. [10], (based on a routine of G. Passarino), the input value gh is not used,

sgh=2 : gh is calculated according to the complex-pole scheme [10] (using cpHTO11.f by G. Passarino) (default), the input value gh is not used.

gh=0.00421d0 : double-precision number for the Higgs-boson width (default for $M_{\mathrm{H}}=126 \mathrm{GeV}$ ).

Hbr=1d0 : double-precision number for the branching ratio for Higgs decay into singlets. Hbr simply rescales the complete cross section.

If the Higgs decay into singlets is included, the following cuts on the decay products $d_{1,2}$ of the Higgs boson are available:

ptcut $($ decp $)=0 \mathrm{~d} 0 \quad$ : lower $p_{\mathrm{T}}$ cut on Higgs-decay products $d_{1,2}$, $\operatorname{ycut}(\operatorname{decp})=1 \mathrm{~d} 10$ : upper $|y|$ cut on Higgs-decay products $d_{1,2}$.

higgsbetweenjets : integer that dictates whether the event is cut if Higgs- 
decay products $d_{1,2}$ are not produced in between the tagging jets with respect to rapidity.

$$
\begin{aligned}
& \text { higgsbetweenjets }=0: \text { the selection criterion } \min \left(y\left(j_{1}\right), y\left(j_{2}\right)\right)< \\
& y\left(d_{1,2}\right)<\max \left(y\left(j_{1}\right), y\left(j_{2}\right)\right) \text { is not applied, } \\
& \text { higgsbetweenjets }=1: \text { the selection criterion } \min \left(y\left(j_{1}\right), y\left(j_{2}\right)\right)< \\
& y\left(d_{1,2}\right)<\max \left(y\left(j_{1}\right), y\left(j_{2}\right)\right) \text { is applied. }
\end{aligned}
$$

\subsubsection{Parton distribution functions}

HAWK supports the LHAPDF interface to evaluate parton distribution functions (PDFs) for hadronic cross sections. In addition, a few built-in PDF sets are available:

spdf : integer that determines the usage of PDFs.

spdf=1 : MRST2004 parton distributions are used, spdf=11 : CTEQ6M parton distributions are used, spdf=14 : CTEQ6L1 parton distributions are used, spdf=99 : the LHAPDF interface is used, i.e. the PDF set is selected via pdfname or pdfpath as described in the following.

A photon PDF 60 62], which is needed for the cross-section calculation of partonic processes with photons in the initial state, is only available for spdf=1 (using the MRST2004QED set [60]) or for spdf=99 if appropriate parton distributions are used. This is the case for the default PDF set NNPDF2.3QED [61]. For other PDF sets only quark and gluon PDFs are taken into account. In this case, the prediction for the photon-initiated processes is trivially zero.

To select a PDF set using the LHAPDF interface, there are the following input options:

pdfname=NNPDF23_nlo_as_0118_qed.LHgrid : a string that specifies the name of the PDF set to be used. The corresponding file has to be in the directory PDFsets of the PDF installation. The path to the directory PDFsets has to be set in advance, e.g. by export LHAPATH=/... /share/lhapdf/PDFsets in . bashrc.

pdfpath=' / . ./PDFsets/NNPDF23_nlo_as_0118_qed.LHgrid' : a string that specifies the name of the the PDF set including the full path to the file. Note the apostrophes around the specified pdfpath. 
pdfmember : an integer that specifies the member of the selected family of PDF sets. Usually, pdfmember $=0$ (default) is the central value PDF set. The allowed range of pdfmember depends on the selected PDF set and is provided by LHAPDF.

For PDF uncertainty calculations one can simultaneously calculate additional cross sections for more than one PDF set if one uses LHAPDF with a *. LHgrid set (40 sets will only double the runtime). The result for the cross section (including all the corrections) will be written to the specified output together with the results for the central PDF member. Distributions are only produced for the central member specified by pdfmember. The range of PDF members is set by the following input:

pdfmemberfrom : integer that selects the beginning of the range of evaluated PDF members.

pdfmemberto : integer that selects the end of the range of evaluated PDF members.

The default is pdfmemberfrom $=1$ and pdfmemberto $=0$ which means that only the set selected via pdfmember is evaluated, no additional sets are used. If only a *.LHpdf implementation is available one should not use the simultaneous calculation with different PDF sets but evaluate the different sets sequentially, otherwise the runtime explodes due to repeated PDF initializations.

\subsubsection{Factorization and renormalization scales}

The QCD factorization and renormalization scales and schemes are set by the following input:

sfactqcd : integer that selects the QCD factorization scheme.

sfactqcd=1 : $\overline{\mathrm{MS}}$ factorization is used with respect to QCD (default),

sfactqcd=2 : DIS factorization is used with respect to QCD.

sfactqed : integer that selects the QED factorization scheme.

sfactqed=1 : $\overline{\mathrm{MS}}$ factorization is used with respect to QED, sfactqed=2 : DIS factorization is used with respect to QED (default). 
qcdfacscalefac=1d0 : double-precision number that multiplies the default QCD factorization scale.

qcdrenscalefac=1d0 : double-precision number that multiplies the default QCD renormalization scale.

qedfacscalefac=1d0 : double-precision number that multiplies the default QED factorization scale.

The last three parameters rescale the factorization and renormalization scales of QCD and the factorization scale of QED which are set by default to:

$$
\begin{array}{lll}
M_{\mathrm{W}} & \text { for } \mathrm{VBFH} \text { production } & (\text { selprocess }=0), \\
M_{\mathrm{W}}+M_{\mathrm{H}} & \text { for } \mathrm{WH} \text { production } & (\text { selprocess }=1,2), \\
M_{\mathrm{Z}}+M_{\mathrm{H}} & \text { for } \mathrm{ZH} \text { production } & (\text { selprocess }=3,4) .
\end{array}
$$

Phase-space-dependent (often called dynamical) scale choices are not supported by the current version of HAWK.

\subsubsection{Switches for different $L O / N L O$ contributions}

The following switches can be used to switch on or off the LO contributions and various contributions to the NLO corrections. By default all contributions are taken into account.

sborn : integer that includes/excludes the Born contribution.

sborn=0 : tree-level contributions not included, sborn=1 : tree-level contributions included (default).

sbini : integer that includes/excludes contributions from initial-state b quarks.

sbini=0 : initial-state b-quark contributions not included, sbini=1 : initial-state b-quark contributions included (default).

sbfin : integer that includes/excludes contributions from final-state b quarks.

sbfin=0 : final-state b-quark contributions not included, sbfin=1 : final-state b-quark contributions included (default).

The contributions included/excluded via sbini and sbfin are only calculated at LO, since they contribute only at the per-cent level. 
sew : integer that includes/excludes the EW corrections.

sew $=0$ : EW corrections not included, sew=1 : EW corrections included (default).

spinc : integer that includes/excludes incoming photon contributions.

spinc=0 : incoming photon contributions not included,

spinc=1 : incoming photon contributions included (default), if the chosen PDF set includes a photon PDF (see Section 3.3.6).

sqcd : integer that includes/excludes the QCD corrections.

sqcd=0 : QCD corrections not included, sqcd=1: QCD corrections included (default).

For Higgs production in vector-boson fusion ( switches described in the rest of this section are available for contributions to NLO corrections:

shh2 : integer that includes/excludes 2-loop heavy-Higgs corrections.

shh2=0 : 2-loop heavy-Higgs corrections not included, shh2=1 : 2-loop heavy-Higgs corrections included (default). These contributions are taken from Ref. [63].

sqcddiag : integer that includes/excludes diagonal QCD corrections. sqcddiag=0 : diagonal QCD corrections not included, sqcddiag=1 : diagonal QCD corrections included (default).

sqcdnondiag : integer that includes/excludes non-diagonal QCD corrections.

sqcdnondiag=0 : non-diagonal QCD corrections not included, sqcdnondiag=1 : non-diagonal QCD corrections included (default).

sqcdggfus : integer that includes/excludes interference with gluon-fusion contributions.

sqcdggfus=0 : interference with gluon-fusion contributions not included,

sqcdggfus=1 : interference with gluon-fusion contributions included (default). 
sqcdgsplit : integer that includes/excludes interference with gluon-splitting contributions.

sqcdgsplit=0 : interference with gluon-splitting contributions not included,

sqcdgsplit=1 : interference with gluon-splitting contributions included (default).

The precise definition of these contributions can be found in Ref. [2]. If sch2=0 (see below), the diagonal QCD corrections are always excluded, i.e. sqcddiag has no effect. If schint=0 (see below), the non-diagonal QCD corrections, the interferences with gluon fusion, and gluon splitting are always excluded, i.e. sqcdnondiag, sqcdggfus, and sqcdgsplit have no effect. If shvv>0 (see below), sqcdnondiag, sqcdggfus, sqcdgsplit are set to zero, i.e. these contributions are not supported for anomalous Higgs couplings.

The following switches for VBFH can be used to switch on or off contributions connected to $s$ - or $t$-channel diagrams, squared diagrams, or interferences. By default all contributions are included. Since the exclusion of diagrams is not a physical procedure, you should only use this option if you know what you are doing.

sscha : integer that includes/excludes the $s$-channel contribution.

sscha=0 : $s$-channel contribution not included, sscha=1 : s-channel contribution included (default).

stcha : integer that includes/excludes the $t$-channel and $u$-channel contributions.

stcha $=0: t / u$-channel contributions not included, stcha=1 : $t / u$-channel contributions included (default).

sch2 : integer that includes/excludes contributions due to squared LO diagrams and the corresponding corrections (defined by the fermionnumber flow).

sch2=0 : contributions due to squared diagrams not included, sch2=1: contributions due to squared diagrams included (default).

schint : integer which includes/excludes contributions due to interferences (defined as complement to the squared LO diagrams and their corrections steered by sch2). 
schint $=0$ : contributions due to interferences not included,

schint=1 : contributions due to interferences included (default).

To take only squared $t$-channel (and $u$-channel) diagrams into account, which corresponds to the definition of the total VBFH cross section in Refs. [10 12 ] use sscha $=0$, stcha $=1, \operatorname{sch} 2=1$, and schint=0. QCD contributions are in addition steered by the switches sqcddiag, sqcdnondiag, sqcdggfus, and sqcdgsplit discussed above.

\subsubsection{Anomalous $H V V$ couplings}

HAWK supports predictions with anomalous $\mathrm{H} V V$ couplings for $V=$ W,Z. Our implementation of anomalous couplings follows the (modified) parameterization of Ref. [64]. In addition the Standard Model HVV coupling can be rescaled via $r$ sm. In this parameterization, the different (anomalous) couplings are related to the parameters $d, d b, d t$, and $d t b$ via

$$
\begin{aligned}
& \mathrm{a} 1 \mathrm{hwW}=\mathrm{rsm} M_{\mathrm{W}} / s_{\mathrm{W}} \quad=\mathrm{SM} \text { HWW coupling, } \\
& \mathrm{a} 2 \mathrm{hww}=2 \mathrm{~d} /\left(s_{\mathrm{W}} M_{\mathrm{W}}\right) \quad=2 g_{\mathrm{HWW}}^{(2)} \text {, } \\
& \text { a3hww }=2 \mathrm{dt} /\left(s_{\mathrm{W}} M_{\mathrm{W}}\right) \quad=2 \tilde{g}_{\mathrm{HWw}}^{(2)}, \\
& \text { a1haa }=0 \quad=\mathrm{H} \gamma \gamma \text { like HZZ in SM, } \\
& \mathrm{a} 2 \mathrm{haa}=4\left(\mathrm{~d} s_{\mathrm{W}}^{2}+\mathrm{db} c_{\mathrm{W}}^{2}\right) /\left(2 s_{\mathrm{W}} M_{\mathrm{W}}\right)=4 g_{\mathrm{H} \gamma \gamma} \text {, } \\
& \text { a3haa }=4\left(\operatorname{dt~} s_{\mathrm{W}}^{2}+\operatorname{dtb} c_{\mathrm{W}}^{2}\right) /\left(2 s_{\mathrm{W}} M_{\mathrm{W}}\right)=4 \tilde{g}_{\mathrm{H} \gamma \gamma}, \\
& \text { a1haz }=0 \quad=\mathrm{HZ} \gamma \text { like HZZ in SM, } \\
& \mathrm{a} 2 \mathrm{haz}=-2 c_{\mathrm{W}}(\mathrm{d}-\mathrm{db}) / M_{\mathrm{W}} \quad=2 g_{\mathrm{HZ} \gamma}^{(2)} \text {, } \\
& \text { a3haz }=-2 c_{\mathrm{W}}(\mathrm{dt}-\mathrm{dtb}) / M_{\mathrm{W}} \quad=2 \tilde{g}_{\mathrm{HZ} \gamma}^{(2)} \text {, } \\
& \mathrm{a} 1 \mathrm{hzz}=\mathrm{rsm} M_{\mathrm{W}} /\left(s_{\mathrm{W}} c_{\mathrm{W}}^{2}\right)=\text { SM HZZ coupling, } \\
& \mathrm{a} 2 \mathrm{hzz}=4\left(\mathrm{~d} c_{\mathrm{W}}^{2}+\mathrm{db} s_{\mathrm{W}}^{2}\right) /\left(2 s_{\mathrm{W}} M_{\mathrm{W}}\right)=4 g_{\mathrm{HZZ}}^{(2)}, \\
& \mathrm{a} 3 \mathrm{hzz}=4\left(\operatorname{dt} c_{\mathrm{W}}^{2}+\operatorname{dtb} s_{\mathrm{W}}^{2}\right) /\left(2 s_{\mathrm{W}} M_{\mathrm{W}}\right)=4 \tilde{g}_{\mathrm{HZZ}},
\end{aligned}
$$

where the notation for the coupling constants corresponds to the Feynman rule

$$
\text { i a1hvv } g_{\mu \nu}+\mathrm{i} \text { a2hvv }\left(-k_{1} \cdot k_{2} g_{\mu \nu}+k_{1 \nu} k_{2 \mu}\right)+\mathrm{i} \text { a3hvv } \epsilon_{\rho \sigma \mu \nu} k_{1}^{\rho} k_{2}^{\sigma}
$$

for $\mathrm{H} V_{1}\left(k_{1 \mu}\right) V_{2}\left(k_{2 \nu}\right)$ and the $g_{\mathrm{H} V V}$ 's correspond to the notation in Ref. [64]. Note the sign changes in a2haz and a3haz due to our conventions of SM couplings which follow Refs. [65, 66]. Cosine and sine of the electroweak mixing angle are defined as $c_{\mathrm{W}}=M_{\mathrm{W}} / M_{\mathrm{Z}}$ and $s_{\mathrm{W}}=\sqrt{1-c_{\mathrm{W}}^{2}}$, respectively. The input for the anomalous couplings is governed by the following switch: 
shvv : integer that enables/disables anomalous HVV couplings.

shvv=0 : anomalous HVV couplings disabled (default), shvv=1 : anomalous $H V V$ couplings enabled, input for parameters $\mathrm{d}, \mathrm{db}, \mathrm{dt}, \mathrm{dtb}$ expected, shvv=2 : anomalous $\mathrm{H} V V$ couplings enabled, direct input for a1hww, ..., a3hzz expected.

According to shvv, one either specifies d, db, etc. or a1hww, a2hww, etc. as double-precision numbers. By default, all anomalous couplings are set to zero. One can also specify rsm which by default is set to one.

The anomalous couplings to the neutral gauge bosons are switched off for small momentum transfer with a form factor $\left|s_{1}\right|\left|s_{2}\right| /\left(m_{0}^{2}+\left|s_{1}\right|\right) /\left(m_{0}^{2}+\left|s_{2}\right|\right)$ to avoid IR singularities from anomalous couplings, where $s_{1}$ and $s_{2}$ are the virtualities of the two intermediate $\mathrm{W}$ and $\mathrm{Z}$ bosons and $m_{0}=1 \mathrm{GeV}$ is used.

If shvv $>0$, sqcdnondiag, sqcdggfus, and sqcdgsplit are set to zero. These QCD corrections (which are tiny in the SM) are not supported for anomalous Higgs couplings.

To control the scale dependence of the anomalous couplings, there is the option to use a form factor:

lambdahvv $=-2 \mathrm{~d} 0$ : double-precision number that sets the mass scale in the form factor for anomalous couplings in $\mathrm{GeV}$.

lambdahvv $>0$ : form factor $=$ lambdahvv $4 /\left(\right.$ lambdahvv $\left.^{2}+\left|s_{1}\right|\right) /\left(\right.$ lambdahvv $\left.^{2}+\left|s_{2}\right|\right)$,

lambdahvv $<0$ : form factor $=1$ (formal limit lambdahvv $\rightarrow \infty$ ).

\subsubsection{Technical parameters for the Monte Carlo integration}

In order to produce statistically independent HAWK results, one has to use independent random-number sets which can be obtained with the following input:

ranluxseed=0 : integer that yields different random number seeds by choosing different positive integers.

\subsubsection{Parameters steering the output}

The level of detail of the output generated by HAWK is steered by the following switches:

Inoutmc : integer that determines the level of detail of the HAWK output. 
lnoutmc=1: standard output of Monte Carlo,

lnoutmc $=3$ : intermediate output of Monte Carlo,

lnoutmc=5 : full output of Monte Carlo.

shisto : integer that determines the generation of histograms for differential distributions.

shisto $=0$ : no histograms are produced, shisto=1 : histograms are produced (default).

By default, histograms for standard hadron-collider observables relevant for the selected Higgs-production process are generated. Additional histograms can be implemented by adapting the subroutines settings_Hjj in vbfh_public.f or the subroutines settings_Hll, settings_Hlv, settings_Hvl, or settings_Hvv in whzh_public.f depending on selprocess.

\subsection{Parallel execution using the MPI standard}

HAWK supports parallel execution using MPI. To use the parallel version set $F C=\$(M P I F C)$ in the Makefile, where MPIFC should be your MPI Fortran compiler. The parallel version of HAWK has been tested using the Intel Fortran compiler 11.1 and SUN's MPI 8.2. Using HAWK with MPI, the input cannot be provided via standard input $(. /$ hawk-2.0 < inputfile), but has to be put into a file with the literal name inputfile in the directory of the hawk-2.0 executable.

\section{HAWK output and sample runs}

By default, all output is written to standard output. An outputfile can, however, be specified in the inputfile (see Section 3.3).

The output includes the process that has been selected via selprocess and the SM parameters which have been used. The values for anomalous couplings and information on the treatment of an off-shell Higgs boson are provided if relevant (see Section [3.3). In addition, information about the Monte Carlo integration is provided, including the number of events generated during the run, collider information, the PDF choice, scale choices, included contributions, calculated corrections, the jet algorithm, and applied cuts. Information on the seed for the random numbers is given as well.

HAWK provides intermediate results for monitoring the progress of the Monte Carlo integration. The central final result for the total cross section can be found under 


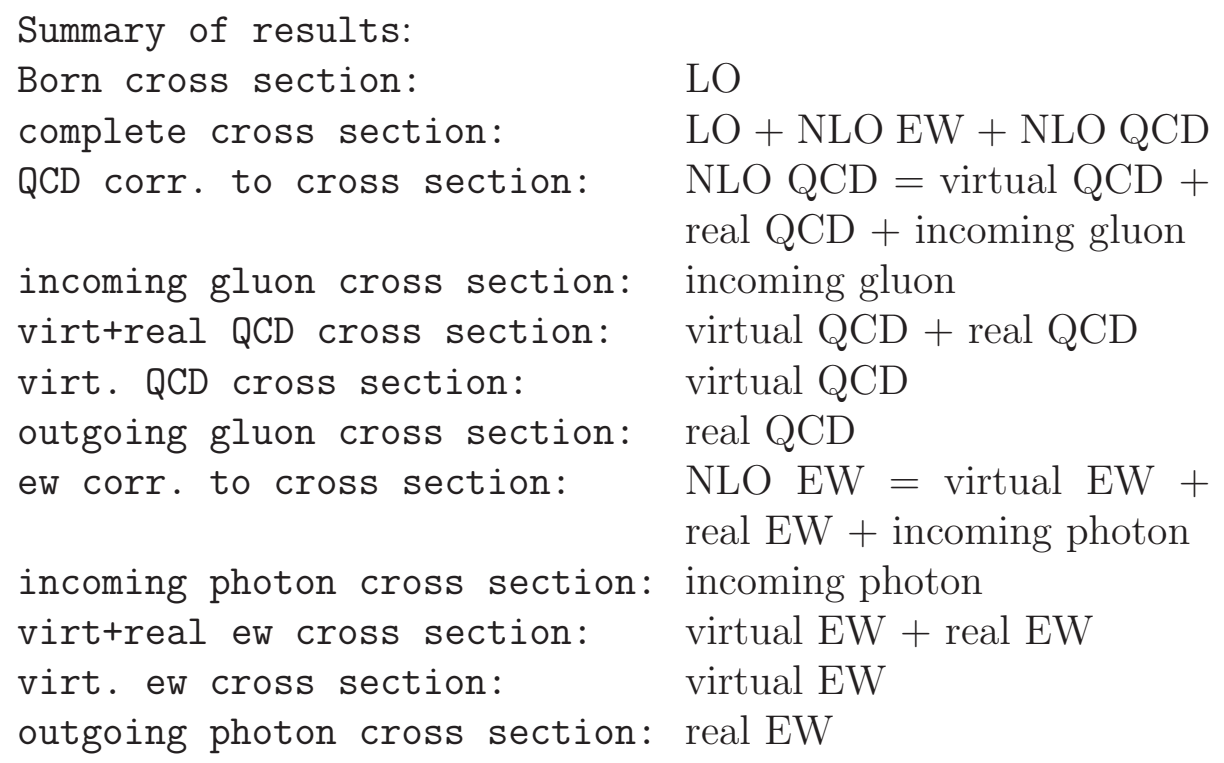

All results for cross sections are given in femtobarns (fb). In addition, the relative corrections, normalized to the LO cross section, are provided.

\subsection{Differential distributions with HAWK}

For shisto=1, the program produces a set of histograms for differential distributions of standard hadron-collider observables. The produced histograms are listed at the end of the HAWK output together with the range of the binned differential observable. The distributions which are available independently of the selected process are written to the files

dat.pth distribution in transverse momentum of Higgs,

dat.yh distribution in rapidity of Higgs,

dat.etah distribution in pseudo-rapidity of Higgs.

In addition, for shtr $>0$, HAWK also provides

$$
\begin{array}{ll}
\text { dat.mh } & \text { distribution in invariant mass of off-shell Higgs boson, } \\
& \text { centered around the Higgs-boson mass, } \\
\text { dat.mhwide } & \text { distribution in invariant mass of off-shell Higgs boson } \\
& \text { in a wide range. }
\end{array}
$$

For VBFH production, i.e. selprocess $=0$, the following jet distributions are 
available,

$\begin{array}{ll}\text { dat.ptjmax1pt } & \text { distribution in transverse momentum of leading } \\ & \text { jet } j_{1}, \\ \text { dat.ptjmax2pt } & \text { distribution in transverse momentum of sublead- } \\ & \text { ing jet } j_{2}, \\ \text { dat.yjmax1pt } & \text { distribution in rapidity of leading jet } j_{1}, \\ \text { dat.yjmax2pt } & \text { distribution in rapidity of subleading jet } j_{2}, \\ \text { dat.dyjjmaxpt } & \text { distribution in rapidity difference of leading jets, } \\ \text { dat.dphijjmaxpt } & \text { distribution in azimuthal angle difference of } \\ & \text { leading jets, } \\ \text { dat.mjjmaxpt } & \text { distribution in invariant mass of leading jets, }\end{array}$

where the leading and subleading jets are ordered in $p_{\mathrm{T}}$.

For the Higgs-strahlung processes, selprocess $>0$, HAWK provides

dat.ptv distribution in transverse momentum of the vector boson $\left(\mathrm{W}^{ \pm}\right.$or $\left.\mathrm{Z}\right)$

and the following distributions concerning the final-state leptons

$\begin{array}{ll}\text { dat.ptlp } & \text { distribution in transverse momentum of } l^{+}, \\ \text {dat.ylp } & \text { distribution in rapidity of } l^{+}, \\ \text {dat.etalp } & \text { distribution in pseudo-rapidity of } l^{+}, \\ \text {dat.ptmiss } & \text { distribution in transverse momentum of } \nu\left(=p_{\mathrm{T}, \mathrm{miss}}\right) \text {, } \\ \text { dat.ymiss } & \text { distribution in rapidity of } \nu, \\ \text { dat.etamiss } & \text { distribution in pseudo-rapidity of } \nu, \\ \text { dat.dphilpH } & \text { distribution in azimuthal angle difference of } l^{+} \text {and } \\ & \text { Higgs }\end{array}$

for $\mathrm{W}^{+} \mathrm{H}$ production. The analogous distributions are available for $\mathrm{W}^{-} \mathrm{H}$ production ( $\mathrm{lp}$ is replaced by $\mathrm{Im}$ for the $l^{-}$distributions). For $\mathrm{ZH}$ production with Z-boson decay into charged leptons, the distributions are of course available for the negatively and the positively charged lepton. For ZH production with Z-boson decay into neutrinos, the distributions for missing transverse momentum refer to the sum of the neutrino momenta.

The relative EW corrections $\delta_{\mathrm{EW}}(\mathcal{O})$ for a bin-by-bin differential reweighting, as suggested in Section 2.4, are obtained by the ratio of entries in the columns Monte Carlo average for virt+real ew cross-section and 
Monte Carlo average for the Born cross-section in each bin as provided in the text file for a given distribution. The contributions due to photons in the initial state are given in the column Monte Carlo average for incoming photon cross-section.

The histograms can be adapted, and other histograms can be included by modifying the respective subroutine settings_Hxx in vbfh_public.F or whzh_public.F, respectively.

For the histograms in Ref. [2] $10^{9}$ events were used, and the histograms were rebinned to 20 bins.

\subsection{Sample runs}

We provide several sample input files along with the corresponding results. They can be found in the corresponding subdirectories of HAWK-2.0/sampleruns. All sample runs (apart from input_default) use $10^{7}$ events and default input parameters and cuts for the corresponding processes. They typically take between 0.5 hours (testrun. Hnn) to 2.5 hours (testrun.Hjj_ofs) (with the ifort compiler, with gfortran run times are typically a factor $2-3$ larger). The following sample runs are available:

input_default:

This file shows all input parameters with their default values and provides a run with $10^{6}$ events for VBFH, pp $\rightarrow \mathrm{H}+2$ jets, for on-shell Higgs boson, taking about 15 minutes (with the ifort compiler). The output can be found in output_default. The file input_sample is equivalent to input_default, making use of the default settings in HAWK.

input_samplerun. $\mathrm{Hj} \mathrm{j}$ :

sample run for $\mathrm{VBFH}, \mathrm{pp} \rightarrow \mathrm{H}+2$ jets, for on-shell Higgs boson.

input_samplerun.Hjj.ofs:

sample run for VBFH, pp $\rightarrow \mathrm{H}+2$ jets, for off-shell Higgs boson using an off-shell propagator.

input_samplerun. Hmm:

sample run for pp $\rightarrow \mathrm{HZ} \rightarrow \mathrm{H} \mu^{+} \mu^{-}$, for bare muons and on-shell Higgs boson.

input_samplerun.Hnn:

sample run for $\mathrm{pp} \rightarrow \mathrm{HZ} \rightarrow \mathrm{H} \bar{\nu}_{\mathrm{e}} \nu_{\mathrm{e}}$, for on-shell Higgs boson. 
input_samplerun.Hmn:

sample run for $\mathrm{pp} \rightarrow \mathrm{HW}^{+} \rightarrow \mathrm{H} \mu^{+} \nu_{\mathrm{e}}$, for bare anti-muon and on-shell Higgs boson.

input_samplerun. Hnm:

sample run for pp $\rightarrow \mathrm{HW}^{-} \rightarrow \mathrm{H} \bar{\nu}_{\mathrm{e}} \mu^{-}$, for bare muon and on-shell Higgs boson.

input_samplerun.Hmn.undet_lepton:

sample run for $\mathrm{pp} \rightarrow \mathrm{HW}^{+} \rightarrow \mathrm{H} \mu^{+} \nu_{\mathrm{e}}$, for on-shell Higgs boson and undetected bare anti-muon.

input_samplerun.Hjj.ac:

sample run for $\mathrm{VBFH}, \mathrm{pp} \rightarrow \mathrm{H}+2$ jets, for on-shell Higgs boson with anomalous HVV couplings.

input_samplerun.Hmn.ac:

sample run for $\mathrm{pp} \rightarrow \mathrm{HW}^{+} \rightarrow \mathrm{H} \mu^{+} \nu_{\mathrm{e}}$, for bare anti-muon and on-shell Higgs boson with anomalous HVV couplings.

\section{Conclusions}

The Monte Carlo program HAWK provides predictions for Higgs-boson production via weak vector-boson fusion or Higgs strahlung ( $V H$ production) at hadron colliders, including the full set of next-to-leading order corrections of the strong and electroweak interactions. In $V H$ production, leptonic decays of the weak gauge boson $V=\mathrm{W}, \mathrm{Z}$ and the corresponding off-shell effects are included at this level of accuracy. Off-shell effects of the Higgs boson can be simulated as well, but only for vector-boson fusion a decay into a pair of massless singlets is supported in the current version.

Predictions are provided in the framework of the Standard Model, optionally extended by anomalous Higgs-gauge-boson interactions. Further generalizations of the program to models with extended Higgs sectors are planned for future updates of HAWK.

\section{Acknowledgements}

The work of A.D. was partially supported by the Bundesministerium für Bildung und Forschung (BMBF) under under contract no. 05H12WWE. 
[1] M. Ciccolini, A. Denner and S. Dittmaier, Phys. Rev. Lett. 99 (2007) 161803 arXiv:0707.0381 [hep-ph]].

[2] M. Ciccolini, A. Denner and S. Dittmaier, Phys. Rev. D77 (2008) 013002 [arXiv:0710.4749 [hep-ph]].

[3] A. Denner, S. Dittmaier, S. Kallweit and A. Mück, JHEP 1203 (2012) 075 arXiv:1112.5142 [hep-ph]].

[4] S. Chatrchyan et al. [CMS Collaboration], Phys. Lett. B 716 (2012) 30 arXiv:1207.7235 [hep-ex]].

[5] G. Aad et al. [ATLAS Collaboration], Phys. Lett. B 716 (2012) 1 arXiv:1207.7214 [hep-ex]].

[6] S. Chatrchyan et al. [CMS Collaboration], Phys. Rev. D 89 (2014) 092007 arXiv:1312.5353 [hep-ex]].

[7] S. Chatrchyan et al. [CMS Collaboration], Phys. Rev. Lett. 110 (2013) 081803 [arXiv:1212.6639 [hep-ex]].

[8] G. Aad et al. [ATLAS Collaboration], Phys. Lett. B 726 (2013) 88 arXiv:1307.1427 [hep-ex]].

[9] G. Aad et al. [ATLAS Collaboration], Phys. Lett. B 726 (2013) 120 arXiv:1307.1432 [hep-ex]].

[10] S. Dittmaier, C. Mariotti, G. Passarino and R. Tanaka et al. [LHC Higgs Cross Section Working Group Collaboration], CERN-2011-002, arXiv:1101.0593 [hep-ph].

[11] S. Dittmaier, C. Mariotti, G. Passarino and R. Tanaka et al. [LHC Higgs Cross Section Working Group Collaboration], CERN-2012-002, arXiv:1201.3084 [hep-ph].

[12] S. Heinemeyer, C. Mariotti, G. Passarino and R. Tanaka et al. [LHC Higgs Cross Section Working Group Collaboration], CERN-2013-004, arXiv:1307.1347 [hep-ph].

[13] A. Denner, S. Dittmaier, S. Kallweit and A. Mück, HAwK, http://omnibus.uni-freiburg.de/sd565/programs/hawk/hawk.html. 
[14] V. D. Barger, R. J. N. Phillips and D. Zeppenfeld, Phys. Lett. B 346 (1995) 106 hep-ph/9412276.

[15] D. L. Rainwater and D. Zeppenfeld, JHEP 9712 (1997) 005 hep-ph/9712271].

[16] D. L. Rainwater, D. Zeppenfeld and K. Hagiwara, Phys. Rev. D 59 (1999) 014037 hep-ph/9808468.

[17] D. L. Rainwater and D. Zeppenfeld, Phys. Rev. D 60 (1999) 113004 [Erratum-ibid. D 61 (2000) 099901] [hep-ph/9906218].

[18] V. Del Duca et al., JHEP 0610 (2006) 016 hep-ph/0608158.

[19] P. Bolzoni, F. Maltoni, S. O. Moch and M. Zaro, Phys. Rev. Lett. 105 (2010) 011801 arXiv:1003.4451 [hep-ph]].

[20] P. Bolzoni, F. Maltoni, S. O. Moch and M. Zaro, Phys. Rev. D 85 (2012) 035002 [arXiv:1109.3717 [hep-ph]].

[21] M. Spira, Fortsch. Phys. 46 (1998) 203 [hep-ph/9705337].

[22] T. Han, G. Valencia and S. Willenbrock, Phys. Rev. Lett. 69 (1992) 3274 [hep-ph/9206246].

[23] T. Figy, C. Oleari and D. Zeppenfeld, Phys. Rev. D 68 (2003) 073005 hep-ph/0306109].

[24] J. Baglio et al., arXiv:1404.3940 [hep-ph].

[25] P. Nason and C. Oleari, JHEP 1002 (2010) 037 arXiv:0911.5299 [hep$\mathrm{ph}]]$.

[26] J. Alwall et al., JHEP 1407 (2014) 079 [arXiv:1405.0301 [hep-ph]].

[27] T. Figy, S. Palmer and G. Weiglein, JHEP 1202 (2012) 105 arXiv:1012.4789 [hep-ph]].

[28] O. Brein, A. Djouadi and R. Harlander, Phys. Lett. B579 (2004) 149 [hep-ph/0307206].

[29] R. Hamberg, W. L. van Neerven and T. Matsuura, Nucl. Phys. B359 (1991) 343. 
[30] O. Brein, R. Harlander, M. Wiesemann and T. Zirke, Eur. Phys. J. C 72 (2012) 1868 arXiv:1111.0761 [hep-ph]].

[31] O. Brein, R. V. Harlander and T. J. E. Zirke, Comput. Phys. Commun. 184 (2013) 998 [arXiv:1210.5347 [hep-ph]].

[32] G. Ferrera, M. Grazzini and F. Tramontano, arXiv:1107.1164 [hep-ph].

[33] G. Ferrera, M. Grazzini and F. Tramontano, arXiv:1407.4747 [hep-ph].

[34] T. Han and S. Willenbrock, Phys. Lett. B273 (1991) 167;

H. Baer, B. Bailey and J. F. Owens, Phys. Rev. D47 (1993) 2730;

J. Ohnemus and W. J. Stirling, Phys. Rev. D47 (1993) 2722.

[35] M. Spira, V2HV, http://people.web.psi.ch/spira/v2hv, 2007.

[36] J. Campbell and K. Ellis, MCFM - Monte Carlo for FeMtobarn processes, http://mcfm.fnal.gov, 2010.

[37] K. Hamilton, P. Richardson and J. Tully, JHEP 0904 (2009) 116 arXiv:0903.4345 [hep-ph]].

[38] G. Luisoni, P. Nason, C. Oleari and F. Tramontano, JHEP 1310 (2013) 083 [arXiv:1306.2542 [hep-ph]].

[39] M. L. Ciccolini, S. Dittmaier and M. Krämer, Phys. Rev. D68 (2003) 073003 [hep-ph/0306234].

[40] L. Altenkamp, S. Dittmaier, R. V. Harlander, H. Rzehak and T. J. E. Zirke, JHEP 1302 (2013) 078 [arXiv:1211.5015 [hep-ph]].

[41] A. Denner, S. Dittmaier, M. Roth and D. Wackeroth, Nucl. Phys. B 560 (1999) 33 [hep-ph/9904472].

[42] A. Denner, S. Dittmaier, M. Roth and L. H. Wieders, Nucl. Phys. B 724 (2005) 247 [Erratum-ibid. B 854 (2012) 504] hep-ph/0505042].

[43] J. Küblbeck, M. Böhm and A. Denner, Comput. Phys. Commun. 60 (1990) 165;

H. Eck and J. Küblbeck, Guide to FeynArts 1.0, University of Würzburg, 1992. 
[44] T. Hahn, Comput. Phys. Commun. 140 (2001) 418 hep-ph/0012260]; T. Hahn and C. Schappacher, Comput. Phys. Commun. 143 (2002) 54 hep-ph/0105349].

[45] T. Hahn and M. Pérez-Victoria, Comput. Phys. Commun. 118 (1999) 153 hep-ph/9807565.

[46] S. Dittmaier, Phys. Rev. D 59 (1999) 016007 hep-ph/9805445].

[47] G. Passarino and M. Veltman, Nucl. Phys. B 160 (1979) 151.

[48] A. Denner and S. Dittmaier, Nucl. Phys. B 658 (2003) 175 hep-ph/0212259.

[49] A. Denner and S. Dittmaier, Nucl. Phys. B 734 (2006) 62 hep-ph/0509141].

[50] A. Denner and S. Dittmaier, Nucl. Phys. B844 (2011) 199 arXiv:1005.2076 [hep-ph]].

[51] S. Dittmaier and M. Roth, Nucl. Phys. B 642 (2002) 307 hep-ph/0206070].

[52] A. Denner, S. Dittmaier, M. Roth and D. Wackeroth, Comput. Phys. Commun. 153 (2003) 462 [hep-ph/0209330].

[53] S. Catani and M. H. Seymour, Nucl. Phys. B 485 (1997) 291 [Erratumibid. B 510 (1998) 503] hep-ph/9605323).

[54] S. Dittmaier, A. Kabelschacht and T. Kasprzik, Nucl. Phys. B 800 (2008) 146 [arXiv:0802.1405 [hep-ph]].

[55] S. Dittmaier, Nucl. Phys. B 565 (2000) 69 [hep-ph/9904440].

[56] A. Buckley, New access to PDF data via LHAPDF6 in Les Houches 2013: Physics at TeV Colliders: Standard Model Working Group Report, eds. J. Butterworth et al., arXiv:1405.1067.

[57] G. C. Blazey et al., in QCD and Weak Boson Physics in Run II, eds. U. Baur, R.K. Ellis and D. Zeppenfeld (Fermilab-Pub-00/297, Fermilab, 2000) p. 47, hep-ex/0005012. 
[58] T. Figy and D. Zeppenfeld, Phys. Lett. B 591 (2004) 297 hep-ph/0403297.

[59] C. Anastasiou, S. Buehler, F. Herzog and A. Lazopoulos, JHEP 1112 (2011) 058 [arXiv:1107.0683 [hep-ph]].

[60] A. D. Martin, R. G. Roberts, W. J. Stirling and R. S. Thorne, Eur. Phys. J. C 39 (2005) 155 [hep-ph/0411040.

[61] S. Carrazza [NNPDF Collaboration], PoS DIS 2013 (2013) 279 arXiv:1307.1131 [hep-ph]].

[62] H. Spiesberger, Phys. Rev. D 52 (1995) 4936 [hep-ph/9412286];

M. Roth and S. Weinzierl, Phys. Lett. B590 (2004) 190 hep-ph/0403200].

[63] A. Frink, B. A. Kniehl, D. Kreimer and K. Riesselmann, Phys. Rev. D 54 (1996) 4548 hep-ph/9606310].

[64] V. Hankele, G. Klämke, D. Zeppenfeld and T. Figy, Phys. Rev. D 74 (2006) 095001 hep-ph/0609075].

[65] M. Böhm, H. Spiesberger and W. Hollik, Fortsch. Phys. 34 (1986) 687.

[66] A. Denner, Fortsch. Phys. 41 (1993) 307 arXiv:0709.1075 [hep-ph]]. 